and long-term immunological and clinical benefits of this approach need to be thoroughly investigated.

\section{Acknowledgments}

We thank Rogier van Gent, Mette Hazenberg, and Jose Borghans for their input.

Address correspondence to: Frank Miedema, University Medical Center Utrecht, Department of Immunology, Lundlaan 6, PO Box 85090, Utrecht UNK 3508 AB, The Netherlands. Phone: 31-88-755-7674; Fax: 31-30-250-4305; E-mail: f.miedema@ umcutrecht.nl.

1. Taub, D.D., and Longo, D.L. 2005. Insights into thymic aging and regeneration. Immunol. Rev. 205:72-93.

2. Clark, D.R., De Boer, R.J., Wolthers, K.C., and Miedema, F. 1999. T cell dynamics in HIV-1 infection. Adv. Immunol. 73:301-327.
3. Hakim, F.T., et al. 2005. Age-dependent incidence, time course, and consequences of thymic renewal in adults. J. Clin. Invest. 115:930-939.

4. Berzins, S.P., Boyd, R.L., and Miller, J.F.A.P. 1998. The role of the thymus and recent thymic migrants in the maintenance of the adult peripheral lymphocyte pool. J. Exp. Med. 187:1839-1848.

5. Hazenberg, M.D., Borghans, J.A., De Boer, R.J., and Miedema, F. 2003. Thymic output: a bad TREC record. Nat. Immunol. 4:97-99.

6. Fry, T.J., and Mackall, C.L. 2005. The many faces of IL-7: from lymphopoiesis to peripheral $\mathrm{T}$ cell maintenance. J. Immunol. 174:6571-6576.

7. van den Brink, M.R., Alpdogan, O., and Boyd, R.L. 2004. Strategies to enhance T-cell reconstitution in immunocompromised patients. Nat. Rev. Immunol. 4:856-867.

8. Storek, J., et al. 2003. Interleukin-7 improves CD4 T-cell reconstitution after autologous CD34 cell transplantation in monkeys. Blood. 101:4209-4218.

9. Sportes, C., and Gress, R.E. 2007. Interleukin-7 immunotherapy. Adv. Exp. Med. Biol. 601:321-333.

10. Dixit, V.D., et al. 2007. Ghrelin promotes thymopoiesis during aging. J. Clin. Invest. 117:2778-2790.

11. Murphy, W.J., and Longo, D.L. 2000. Growth hormone as an immunomodulating therapeutic agent.
Immunol. Today. 21:211-213.

12. Wit, J.M., Kooijman, R., Rijkers, G.T., and Zegers, B.J. 1993. Immunological findings in growth hormone-treated patients. Horm. Res. 39:107-110.

13. Napolitano, L.A., et al. 2008. Growth hormone enhances thymic function in HIV-1-infected adults. J. Clin. Invest. 118:1085-1098.

14. Napolitano, L.A., et al. 2002. Increased thymic mass and circulating naive CD4 T cells in HIV-1-infected adults treated with growth hormone. AIDS. 16:1103-1111.

15. Lo, J.C., et al. 2004. The effects of low-dose growth hormone in HIV-infected men with fat accumulation: a pilot study. Clin. Infect. Dis. 39:732-735.

16. Hazenberg, M.D., et al. 2004. Establishment of the CD4+ T-cell pool in healthy children and untreated children infected with HIV-1. Blood. 104:3513-3519.

17. Haynes, B.F., et al. 1999. Analysis of the adult thymus in reconstitution of $\mathrm{T}$ lymphocytes in HIV-1 infection. J. Clin. Invest. 103:453-460.

18. Connors, M., et al. 1997. HIV infection induces changes in $\mathrm{CD}^{+} \mathrm{T}$-cell phenotype and depletions within the $\mathrm{CD} 4^{+} \mathrm{T}$ cell repertoire that are not immediately restored by antiviral or immune-based therapies. Nat. Med. 3:533-540.

\title{
Discovering early molecular determinants of leukemogenesis
}

\author{
Grover C. Bagby \\ Departments of Medicine and Molecular and Medical Genetics, Oregon Health and Sciences University, \\ and Northwest Veterans Affairs Cancer Research Center, Portland, Oregon, USA.
}

\begin{abstract}
Truncating mutations of the G-CSF receptor are found during disease course in nearly half of all patients with severe congenital neutropenia. In this issue of the JCI, Liu et al. demonstrate that these mutations confer a competitive clonal advantage upon HSCs in mice and that the advantage is conditional because it is observed only in the presence of the ligand G-CSF (see the related article beginning on page 946). Once activated, the mutant receptor requires the function of Stat 5 in order to effect clonal expansion of this stem cell population. The results support the notion that early molecular steps in this and other neoplastic processes represent adaptations in which, through somatic mutations, "unfit" stem cells gain a measure of fitness by altering their relationships with their microenvironment.
\end{abstract}

Advances in biotechnology and genomics have catalyzed enormously important discoveries in the field of cancer biology. Some of the findings from studies of molecular pathogenesis have led to the development of new therapeutic agents that have notably controlled the growth of malignant

Nonstandard abbreviations used: AML, acute myeloid leukemia; ELA2, elastase 2; FA, Fanconi anemia; MDS, myelodysplastic syndrome; SCN, severe congenital neutropenia.

Conflict of interest: The author has declared that no conflict of interest exists.

Citation for this article: J. Clin. Invest. 118:847-850 (2008). doi:10.1172/JCI35109. cells in vivo (1). Truly targeted therapy is that which effectively interdicts a survival or replication pathway on which the malignant cell depends but normal cells do not. To develop this kind of therapeutic agent requires that one first identify such a defect in a malignant cell population, then develop an agent that attacks it in a specific way. The target must also be validated in clinical trials. That is, clinical responses must be attributable to the capacity of the therapeutic agent to interdict the function of the target molecule in the neoplastic cells.

Not only have advances in molecularly targeted therapy saved thousands of lives
(1), the successes to date have validated the simple principle that if you understand it you can fix it. For cancer therapeutics this idea is widely accepted today and is supported by massive investments in this approach by pharmaceutical and biotechnology firms. However, while few would argue with the idea that preventing cancer is better than treating it, investigators in the field of cancer prevention have not particularly warmed to the notion that the molecular strategies used for developmental therapeutics can support their goals as well. This situation has to change because there is clear-cut experimental evidence that the earliest control points for carcinogenic change in stem cells can be identified by focusing on stem cell pools under stress. The natural implication is that by interdicting this stress one might prevent the evolution of neoplastic clones.

\section{Stressed stem cell pools are vulnerable to neoplastic change}

Most leukemias evolve as clonal outgrowths of single, somatically mutated HSCs. Studies of patients with inherited 
Table 1

Risk of neoplastic complications in patients with inherited bone marrow failure syndromes

$\begin{array}{lcc}\text { Disease } & \text { Risk of AML/MDS } & \begin{array}{c}\text { Cumulative probability } \\ \text { of malignant disease }\end{array} \\ \text { FA } & \text { Highly increased } & 85 \% \\ \text { Dyskeratosis congenita } & \text { Highly increased } & 35 \% \\ \text { SCN } & \text { Highly increased } & 55 \% \\ \text { Diamond Blackfan anemia } & \text { Increased } & 52 \% \\ \text { Shwachman-Diamond syndrome } & \text { Increased } & 71 \% \\ \text { Amegakaryocytic thrombocytopenia } & \text { Highly increased } & 53 \% \\ \text { Thrombocytopenia absent radius syndrome } & \text { Increased } & 14 \%\end{array}$

ABy age 40-50 yr; ref. 20.

bone marrow failure syndromes (Table 1), in which the HSCs are either constitutively proapoptotic or hypersensitive to apoptotic cues, suggest that the apoptotic state itself represents a selective pressure that favors the evolution of neoplastic clones. The apoptotic cues that put these "unfit" (in the Darwinian sense) HSCs at risk have not been fully defined in these inherited syndromes, but in the case of the inherited bone marrow failure syndrome Fanconi anemia (FA), sufficient evidence has developed to identify TNF- $\alpha$ as one of the key pathophysiological factors influencing clonal evolution in FA HSCs (2).

Up to $40 \%$ of children and young adults with FA $(3,4)$ will exhibit signs of clonal evolution in the bone marrow, a process in which somatically mutated HSCs selfreplicate and give rise to progeny that overtake the bone marrow, leading to myelodysplastic syndrome (MDS) and acute myeloid leukemia (AML). Prior to the onset of clonal evolution, HSCs and committed progenitor cells from the bone marrow of patients bearing inactivating mutations of two FA complementation group C (FANCC) alleles undergo apoptosis in response to a variety of cytokines (including TNF- $\alpha$ ) at very low doses - doses that have no capacity to suppress proliferation and survival of normal progenitors $(5,6)$. In both murine models and in humans with FA, when clonal progeny of somatically mutated stem cells appear in the marrow, the clones are resistant to TNF- $\alpha(2,7,8)$. These results suggested that clonal progeny have ascended either by selection of preexisting covert mutant stem cells (9) or through adaptive mutations that occur during selective sweeps of TNF- $\alpha$ through the HSC pool.

A few months ago, in this journal, Li et al. (2) reported a model of induced clonal selection in which FA HSCs exposed to
TNF- $\alpha$ gave rise to cytogenetically abnormal neoplastic clones ex vivo in a matter of 4-5 weeks. That is, the progeny of a more fit somatically mutated stem cell replaced those of the less fit FA cells because this single stem cell had adapted to the troublesome apoptotic cue. Our expectation that this general model will hold true for some of the other inherited bone marrow failure syndromes is supported by the report in mice from Liu, Link, and colleagues in this issue of the JCI (10), which suggests that the model is applicable to HSCs in the marrow of patients with the inherited bone marrow failure state known as severe congenital neutropenia (SCN).

\section{Clonal evolution in SCN}

$\mathrm{SCN}$ is a genetically heterogeneous inherited disease frequently associated with mutations of the neutrophil elastase 2 (ELA2) gene (11). Patients are reliably responsive to pharmacological doses of G-CSF, an agent that clearly reduces morbidity and mortality in children with this disease. The molecular mechanisms linking ELA2 mutations to failure of granulopoiesis are unclear, although it has been reported that myeloid precursor cells are highly apoptotic owing to constitutive activation of the unfolded protein response in cells bearing ELA2 mutations $(12,13)$. As is the case with most other inherited bone marrow failure syndromes, the relative risk of AML or MDS is high in SCN (see Table 1). Interestingly, truncating mutations of the G-CSF receptor are common in SCN patients (14) and are positively associated with clonal evolution to AML and MDS (15). It had been known for a number of years that this type of mutation resulted in conditional hyperactivation of the G-CSF receptor and resistance to apoptosis in myeloid cells (16).
It was not known, however, whether these activating mutations of the G-CSF receptor played any role in changing the coefficient of selection, specifically in the HSC pool, an important requirement for early leukemogenic mutations. In their current study, Liu, Link, and colleagues (10) used a murine model in which HSCs expressed a truncating mutation of the G-CSF receptor (also known as Csf3r) to demonstrate that exogenous G-CSF permitted the mutant clone to dominate in competitive repopulation experiments. The G-CSF receptor possessing the mutation was not capable of functioning autonomously. That is, exogenous G-CSF was absolutely required. These authors also found that the transcription factor Stat5, known to be activated in normal cells by G-CSF, was absolutely required for the mutant HSCs to be efficient competitive repopulators, a finding that itself clarifies a number of prior uncertainties on the role of STAT5 in HSCs. Clones bearing the mutant receptor did not evolve to AML or MDS in the current study; a finding that recapitulates the human disease in which even highly clonal hematopoiesis does not inevitably progress to leukemia (15). Therefore, studies on the evolution of this mutation in humans and the obvious consequences of the mutation in mice strongly suggest that the G-CSF receptor mutation is an early event in leukemogenesis.

\section{The role of HSC damage in clonal evolution}

The results of this nicely designed study (10) begin to place clonal evolution in SCN in the same category as clonal evolution in FA. In both conditions HSCs are highly apoptotic, a perfect microenvironmental setting for the selection of stem cell clones that have, as a result of somatic mutations or epigenetic changes, acquired resistance to factors in the microenvironment that injure them. In both settings, the somatically mutated HSCs have a high coefficient of selection because the somatic mutation enhanced the relationship of the HSC with its microenvironment. In the case of FA HSCs, new clones escape cell death by interdicting apoptotic responses to TNF- $\alpha$, precisely the environmental cue to which the unevolved FA HSCs are hypersensitive (2). In patients with SCN, neoplastic HSCs emerge as a result of a G-CSF receptor mutation. This implies, of course, that the HSC pool in SCN bone marrow is responding suboptimally to endogenous G-CSF 
Normal hematopoiesis

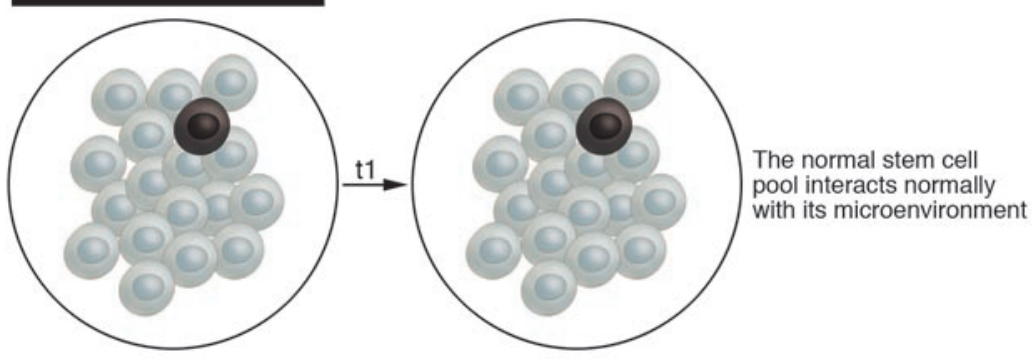

Hereditary bone marrow failure syndromes
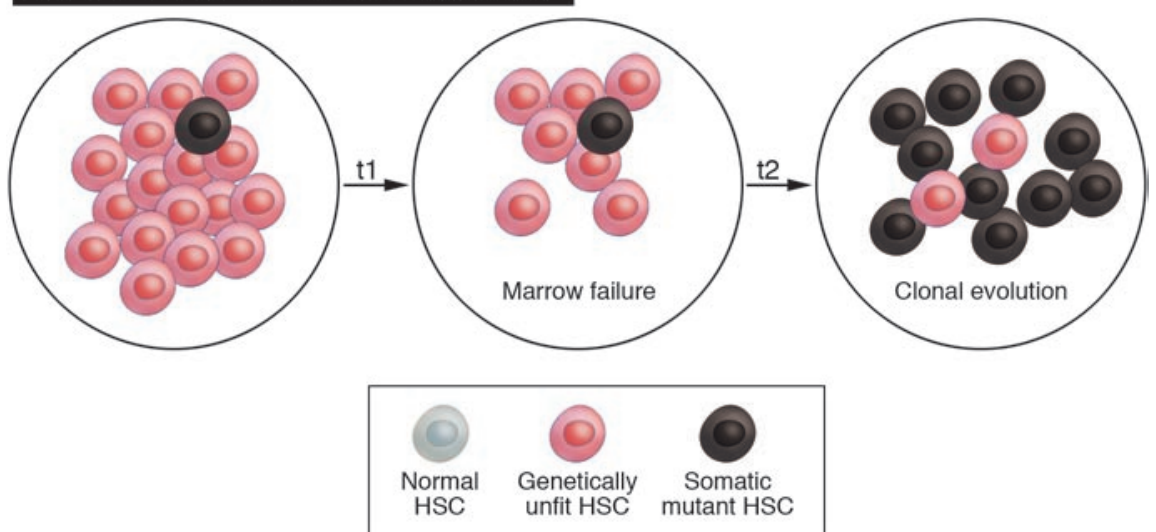

Figure 1

The selection coefficient in HSC pools influences clonal evolution. Potentially leukemogenic translocations can be found in normal individuals who do not go on to develop leukemia (9). Shown here are somatically mutated HSCs (black) in the context of normal bone marrow (normal HSCs are shown in gray) and in the bone marrow of a patient with an inherited bone marrow failure syndrome (genetically unfit HSCs are shown in red). The coefficient of selection for the somatically mutated HSC in normal hematopoiesis is low so the mutant HSC is not significantly more fit. However, when the large population of HSCs is genetically unfit, the same mutant clone has a better opportunity to gain a foothold. Here, the somatically mutated HSCs in the normal and abnormal hematopoietic states have precisely the same mutation. The somatic mutant HSC in the company of the genetically unfit HSCs has a higher selection coefficient, not because of an autonomous attribute, but because the surrounding stem cells are highly unfit. The unfit HSCs are purged from the bone marrow (t1) and are replaced by the progeny of the somatic mutant (this population expands at t2). The data reported in this issue of the $J C /$ by Liu et al. (10) demonstrate that the truncation G-CSF receptor mutation is capable of influencing the expansion of HSCs in the context of G-CSF treatment in mice. Although key studies remain to be performed in human cells, the results do provide a likely pathophysiological mechanism (G-CSF-induced STAT5 activation via a conditionally mutated receptor) for clonal HSC expansion in patients with $\mathrm{SCN}$, expansions that seem to be required very early in the leukemogenic process. t, time.

levels. In SCN and FA the pressure for the emergence of more fit stem cells results in the selection of clones that are better adapted for survival in their respective microenvironments (Figure 1).

The adaptive responses can occur in two ways. Rarely, mutant FA HSCs correct the inherited mutation on one of two mutant alleles. This results in clonal reversion (mosaicism) (17) and, in some cases, complete resolution of bone marrow failure (18). The second pathway of adaptation reflects a kind of molecular "work around" and aberrant activation of antiapoptotic programs. This second adaptive pathway is more maladaptive with respect to the whole patient because the clonal progeny are too resistant to apoptotic cues for their own good. They survive when a normal cell would have reasons not to (for example, when high-level genetic damage should induce apoptotic responses). These mutant cells are more apt to suffer a concatenation of additional mutations and are therefore more likely to evolve to frank leukemic stem cell clones.

The results reported in this issue by Liu et al. (10) help to define some unique investigative opportunities. For example, whether the ELA2 mutation interferes with G-CSF signaling specifically in HSCs should be tested directly. Positive results would explain how the coefficient of selection for G-CSF receptor mutations would be sufficiently high to account for clonal evolution in vivo. Secondly, because activated STAT5 multimerizes and then translocates to the nucleus to function as a transcription factor, experimentally defining promoter binding sites unique to stem cells bearing the G-CSF receptor mutation may help identify gene products that directly enhance stem cell fitness. Third, experiments on clonal progeny that have evolved without a G-CSF receptor mutation might be targeted to factors that modulate G-CSF activity. For example, might loss-of-function mutations involving suppressors of cytokine signaling (19) be involved in these less frequent cases? Most importantly, by defining the full scope and order of adaptive somatic mutations we can begin to contemplate experiments designed to enhance HSC fitness, thereby reducing the likelihood of clonal selection of potentially neoplastic stem cells. It is time to exploit the investigative strategies that have so nicely served developmental therapeutics teams and turn the methods to a bold new aim - molecularly targeted leukemia prevention for patients at high risk.

\section{Acknowledgments}

I thank Laura Hays, Johanna Svahn, Gabrielle Meyers, Qishen Pang, Susan Olson, Robb Moses, Markus Grompe, and D. Wade Clapp for instructive discussions, Susan Bagby for her encouragement, and my laboratory team for their hard work and dedication. I have appreciated the long-standing support of my laboratory by the Department of Veterans Affairs, the NIH, and the OHSU Cancer Institute. I also thank the dedicated staff, founders, executive committee, and scientific advisory board of the Fanconi Anemia Research Fund Inc. for their extraordinary efforts and commitment.

Address correspondence to: Grover C. Bagby, Portland Veterans Affairs Medical Center, Molecular Oncology Research Laboratory, Mailstop: R\&D2, Building 103, Room E221B, 3710 SW Veterans Hospital Road, Portland, Oregon 97239, USA. Phone: (503) 494-0524; Fax: (503) 494-7086; E-mail: grover@ohsu.edu. 
1. Druker, B.J., et al. 2006. Five-year follow-up of patients receiving imatinib for chronic myeloid leukemia. N. Engl. J. Med. 355:2408-2417.

2. Li, J., et al. 2007. TNF-alpha induces leukemic clonal evolution ex vivo in Fanconi anemia group C murine stem cells. J. Clin. Invest. 117:3283-3295.

3. Bagby, G.C., and Meyers, G. 2007. Bone marrow failure as a risk factor for clonal evolution: prospects for leukemia prevention. Hematology. 2007:40-46.

4. Rosenberg, P.S., Greene, M.H., and Alter, B.P. 2003 Cancer incidence in persons with Fanconi anemia. Blood. 101:822-826.

5. Bagby, G.C., and Alter, B.P. 2006. Fanconi anemia. Semin. Hematol. 43:147-156.

6. Haneline, L.S., et al. 1998. Multiple inhibitory cytokines induce deregulated progenitor growth and apoptosis in hematopoietic cells from FAC - / mice. Blood. 91:4092-4098.

7. Lensch, M.W., Rathbun, R.K., Olson, S.B., Jones, G.R., and Bagby, G.C., Jr. 1999. Selective pressure as an essential force in molecular evolution of myeloid leukemic clones: a view from the window of Fanconi anemia. Leukemia. 13:1784-1789.

8. Li, X.X., et al. 2005. Ex vivo culture of Fancc/- stem/ progenitor cells predisposes cells to undergo apop- tosis, and surviving stem/progenitor cells display cytogenetic abnormalities and an increased risk of malignancy. Blood. 105:3465-3471.

9. Mori, H., et al. 2002. Chromosome translocations and covert leukemic clones are generated during normal fetal development. Proc. Natl. Acad. Sci.U. S. A. 99:8242-8247.

10. Liu, F., et al. 2008. Csf $3 r$ mutations in mice confer a strong clonal HSC advantage via activation of Stat5. J. Clin. Invest. 118:946-955.

11. Dale, D.C., et al. 2000. Mutations in the gene encoding neutrophil elastase in congenital and cyclic neutropenia. Blood. 96:2317-2322.

12. Grenda, D.S., et al. 2007. Mutations of the ELA2 gene found in patients with severe congenital neutropenia induce the unfolded protein response and cellular apoptosis. Blood. 110:4179-4187.

13. Kollner, I., et al. 2006. Mutations in neutrophil elastase causing congenital neutropenia lead to cytoplasmic protein accumulation and induction of the unfolded protein response. Blood. 108:493-500.

14. Link, D.C., et al. 2007. Distinct patterns of mutations occurring in de novo AML versus AML arising in the setting of severe congenital neutropenia.
Blood. 110:1648-1655

15. Germeshausen, M., Ballmaier, M., and Welte, K. 2007. Incidence of CSF3R mutations in severe congenital neutropenia and relevance for leukemogenesis: Results of a long-term survey. Blood. 109:93-99.

16. Hunter, M.G., and Avalos, B.R. 2000. Granulocyte colony-stimulating factor receptor mutations in severe congenital neutropenia transforming to acute myelogenous leukemia confer resistance to apoptosis and enhance cell survival. Blood. 95:2132-2137.

17. Lo Ten Foe, J.R., et al. 1997. Somatic mosaicism in Fanconi anemia: Molecular basis and clinical significance. Eur. J. Hum. Genet. 5:137-148.

18. Mankad, A., et al. 2006. Natural gene therapy in monozygotic twins with Fanconi anemia. Blood. 107:3084-3090.

19. Boyle, K., et al. 2007. The SOCS box of suppressor of cytokine signaling-3 contributes to the control of G-CSF responsiveness in vivo. Blood. 110:1466-1474.

20. Alter, B.P. 2007. Diagnosis, genetics, and management of inherited bone marrow failure syndromes. Hematology Am. Soc. Hematol. Educ. Program. 2007:29-39.

\title{
Currying favor for the heart
}

\author{
Jonathan A. Epstein \\ Department of Cell and Developmental Biology, Cardiovascular Institute, and Institute for Regenerative Medicine, \\ University of Pennsylvania, Philadelphia, Pennsylvania, USA.
}

\begin{abstract}
Curcumin, a commonly available spice and alternative medicine, has been tested in the laboratory and the clinic for activity against a wide range of diseases. It is thought to possess antiinflammatory and antioxidant activities and may also function to inhibit histone acetyl transferases, which activate gene expression via chromatin remodeling. Two reports in this issue of the JCI, by Morimoto et al. and $\mathrm{Li}$ et al., suggest that curcumin may inhibit cardiac hypertrophy in rodent models and provide beneficial effects after myocardial infarction or in the setting of hypertension (see the related articles beginning on pages 868 and 879, respectively). These results will spur further mechanistic inquiry into the role of chromatin remodeling in the regulation of cardiac homeostasis.
\end{abstract}

Epigenetics is a term used to describe features of DNA packaging and assembly that modify cellular process and are stably maintained when cells divide, but do not involve changes in DNA sequence. DNA is maintained within the nucleus in an ordered and dynamic structure in association with other proteins, including histones. The complex of DNA and associated proteins is known as chromatin, and an exciting area of active research involves the epigenetic regulation of chro-

Nonstandard abbreviations used: HAT, histone acety transferase; HDAC, histone deacetylase; MEF, myocyte enhancer factor.

Conflict of interest: The author has declared that no conflict of interest exists.

Citation for this article: J. Clin. Invest. 118:850-852 (2008). doi:10.1172/JCI34650. matin structure by enzymes that modify histones. Among these enzymes are those that add or remove acetyl groups on lysine tails of histones. Enzymes that remove acetyl groups are called histone deacetylases (HDACs), and those reactions are reversed by histone acetyl transferases (HATs), which include p300 and CREB-binding protein (CBP). In general, HDACs act as transcriptional repressors, since removal of acetyl groups allows the chromatin to pack more tightly, and access of transcription factors to promoters is restricted. Conversely, HATs tend to function as activators of gene expression.

HDACs have recently been implicated as important regulators of cardiac homeostasis (1). There are at least 11 mammalian HDACs that compose the so-called class
1 and class 2 families, in addition to more distantly related families (2). Inactivation of some of the class 2 HDACs in mice results in cardiac hypertrophy and subsequent heart failure $(1,3,4)$. On the other hand, inactivation of a class 1 HDAC, HDAC2, results in resistance to cardiac hypertrophy (5), which suggests that class 1 and class 2 HDACs may play opposing roles (6). Interestingly, chemical HDAC inhibitors, which block both classes, tend to block hypertrophic responses (6-8). In this issue of the JCI, 2 papers examine the effects of curcumin on the heart and conclude that this commonly available spice blocks HAT activity and prevents cardiac hypertrophy and failure in rodent models $(9,10)$.

\section{Curcumin and cardiac hypertrophy}

Curcumin is a polyphenol responsible for the yellow color of the curry spice turmeric. It has relatively poor bioavailability when taken orally, but also low toxicity (11). It has been touted to possess a myriad of beneficial activities, and clinical trials have been conducted in patients with cancer, rheumatoid arthritis, cystic fibrosis, inflammatory bowel disease, psoriasis, pancreatitis, and other disorders (11-13). Limited data suggest that it possesses antitumor, antioxidant, and antiinflammatory activities. 